\title{
Autonomously functioning thyroid nodule: a patient-based review
}

\author{
M. D. P. Pinto, R. Fernando \\ Department of Surgery, Faculty of Medicine, University of Kelaniya, Sri Lanka
}

Keywords: AFTN; thyrotoxicosis; solitary nodule

\begin{abstract}
Autonomously Functioning Thyroid Nodule (AFTN) is a rare cause of hyperthyroidism. AFTN, first described by Emil Goetsch in 1918, is the presence of a single hyperfunctioning thyroid nodule, which is not under the control of the pituitary/thyroid axis.
\end{abstract}

The current theory on the development of hyperfunctioning nodules is the constitutive activation of Thyroid Stimulating Hormone Receptor (TSHR) due to a somatic point mutation and mutations of Gsa.

The reported incidence of AFTN is about $1 \%$ on the investigation of thyroid nodules. Only about $10 \%-30 \%$ become hyperfunction. Due to the rarity and lack of understanding of its characteristic clinical behaviour, AFTNs are often overlooked and undertreated. The ensuing review is based on a 36-year-old female who underwent left hemithyroidectomy for an AFTN and is well to date.

There are two main issues in the diagnosis and management of AFTN. The first is that the diagnosis is not always made as most patients with AFTNs are functionally euthyroid. The other is the paucity of data in the literature to offer evidencebased individualized management for patients.

As there are no tissue diagnostic criteria for AFTN; clinical, biochemical and radiological assessments to establish TSH independent nodular hyperfunction will clinch the diagnosis. Surgery and Radioiodine ablation remain the main forms of treatment for AFTN. Other methods such as percutaneous ethanol injection therapy (PEIT), laser ablation (LA), radiofrequency ablation (RFA) have a limited role in the management of an AFTN. The newer methods alluded to above will need further evaluation and a better definition of exact roles in the management of an AFTN.

Correspondence: M. D. P. Pinto

E-mail: d.pinto@kln.ac.lk

(iDhttps://orcid.org/0000-0002-3892-2997

Received: 02-07-2021 Accepted: 18-09-2021

DOI: http://doi.org/10.4038/sljs.v39i3.8857

\section{Introduction}

Hyperthyroidism usually refers to increased synthesis and secretion of thyroid hormone from the thyroid gland.

Thyrotoxicosis refers to the clinical syndrome of excess circulating thyroid hormones, due to any cause. The incidence of hyperthyroidism in iodine-sufficient western world is $0.2 \%-1.3 \%$ [1]. The common causes of Hyperthyroidism are diffuse toxic goitre (Graves Disease), toxic nodular goitre (Plummer Disease), and toxic phase of autoimmune thyroiditis. Rarely, an Autonomously Functioning Thyroid Nodule (AFTN) will cause hyperthyroidism. Hyperfunctioning malignant thyroid metastases, drug-induced hyperthyroidism and factitious ingestion of excess thyroid hormone are rare causes of toxic symptoms, especially in the iodine sufficient regions.

AFTN is the presence of a single hyperfunctioning thyroid nodule, which is not under the control of the pituitary/thyroid axis using Thyroid Stimulating Hormone (TSH). AFTN was first described by Emil Goetsch in 1918. Goetsch demonstrated a high concentration of mitochondria in hyperfunctioning thyroid nodules. [2]. In 1960 Tremblay and Pearse did studies and further established the findings of Goetsch. Subsequently further study, AFTN achieved recognition as a separate cause for hyperthyroidism and was known as "Goetsch's disease" by the 1980s [3].

The reported incidence of AFTN is about $1 \%$ on the investigation of thyroid nodules [3]. Most are inert and only about $10 \%$ - 30\% become hyperfunction and cause subclinical or overt thyrotoxicosis [4]. Due to the rarity and lack of understanding of its characteristic clinical behaviour, AFTNs are often overlooked and undertreated.

\section{Case description}

A 36-year-old female had been investigated and treated for a left-sided thyroid nodule for 2 years in another institution. She had been having compressive symptoms such as orthopnoea for 1 year before presentation to our institution. At the onset her illness, her thyroid profile showed a hyperthyroid picture with low TSH - 0.006microIU/ml (0.44.0), high free T3 (fT3) - 5.5pg/ml (1.5-4.1) and normal free T4 (fT4) - 0.871ng/dl (0.8-1.7). An ultrasound scan of the 


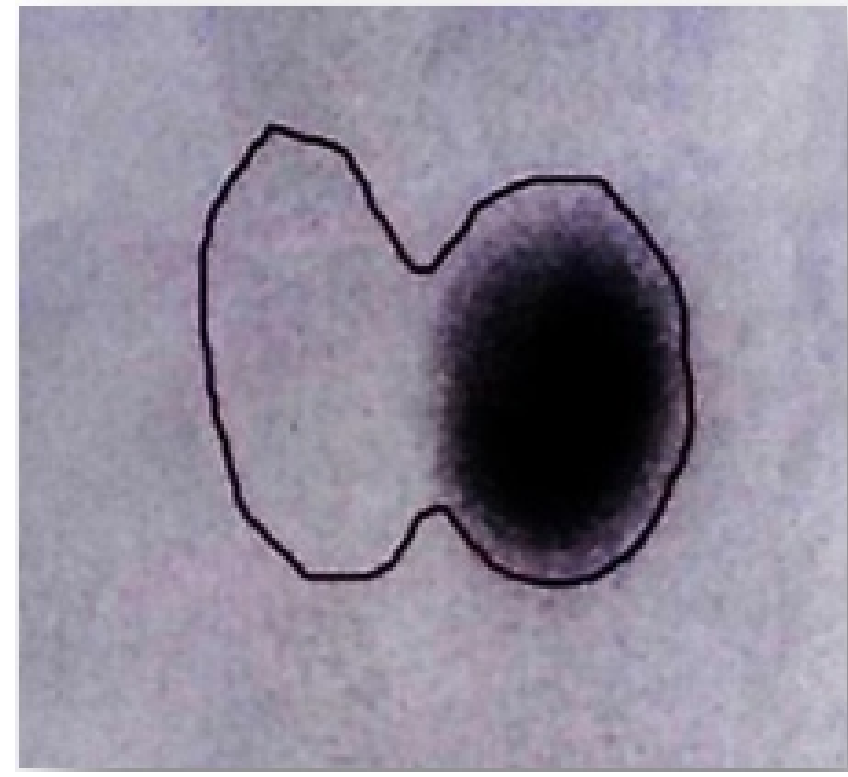

Figure 1. Radioisotope scan of the thyroid gland 20 minutes after injection of $145 \mathrm{mbq}$ of Technetium-99m Pertechnetate

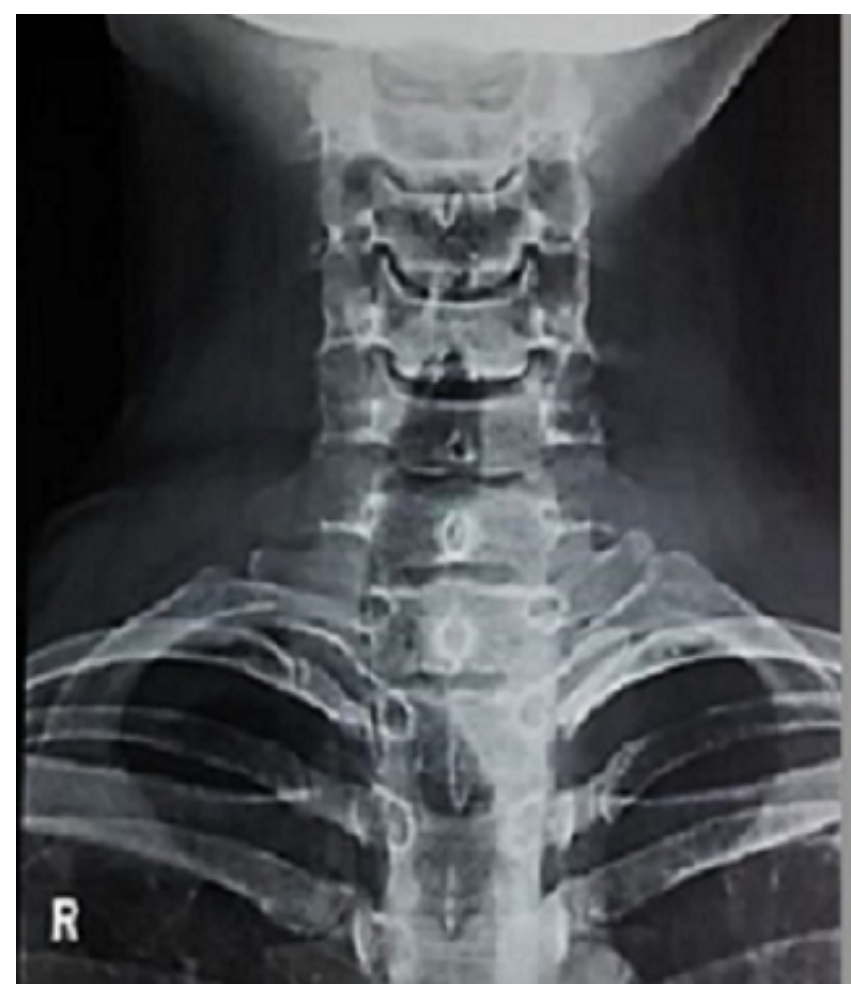

Figure 2. X-Ray of the cervical spine

neck showed a $3.3 \times 2.2 \mathrm{~cm}$ solitary, predominantly cystic nodule in the left lobe with increased vascularity. No calcifications were seen and the rest of the gland plus neck anatomy were unremarkable. Fine Needle Aspiration Cytology (FNAC) showed a Thy 2/ Bethesda II colloid cyst. Radioisotope imaging demonstrated a high uptake in the left lobe with a non-functioning right lobe (Figure 1). She was started on a block and replacement therapy.

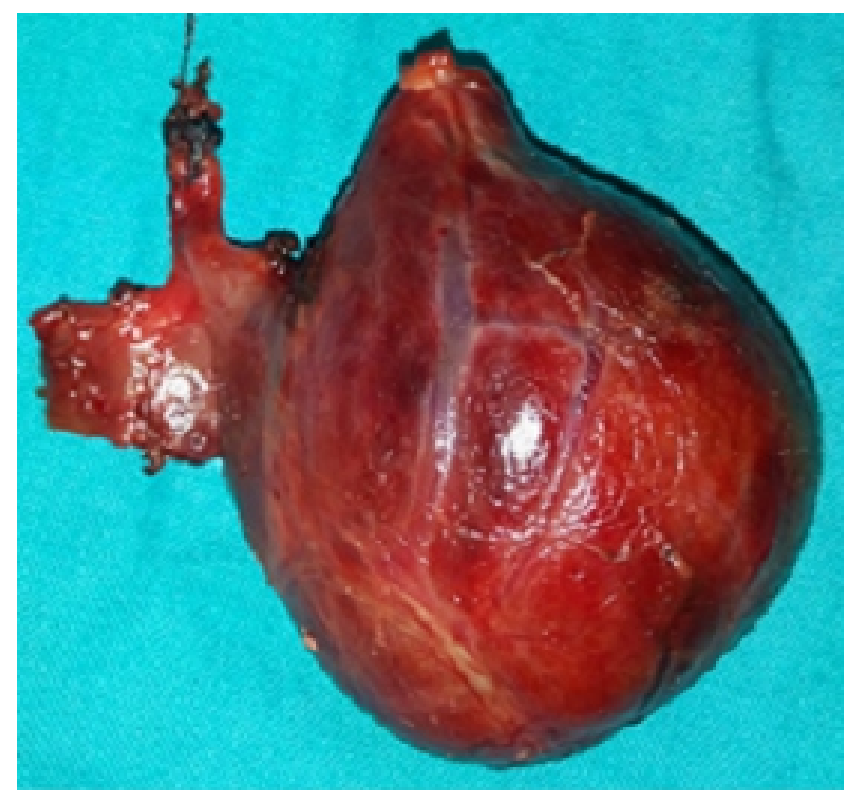

Figure 3. Left hemithyroidectomy specimen

She was referred for consideration of surgery to our unit. At that time she was clinically and biochemically euthyroid. The possibility of a thyroid hemi-agenesis was considered, in the referral, erroneously, due to the appearance of the isotope scan.

The left-sided nodule has grown to $5 \mathrm{~cm}$ in its largest diameter with radiologically benign character. X-Ray of the cervical spine showed mild narrowing and gross shift of the trachea to the right (Figure 2).

Repeat FNAC confirmed a Thy 2 lesion. A diagnosis of an AFTN was made and she underwent left hemithyroidectomy (Figure 3).

A single benign colloid cyst was noted in the histopathological report. Her clinical and biochemical profile postoperatively was euthyroid clinically and biochemically confirming the diagnosis of AFTN. She is well 10 months after surgery and not on thyroxine replacement at present.

\section{Discussion}

A hyperfunctioning/ toxic AFTN or Goetsch's disease is now known as a toxic adenoma. AFTNs occur in every age group with a female preponderance. Toxic AFTNS are common in the elderly ( $>60$ years) [3]. The classical clinical presentation is a single thyroid nodule with features of toxicity.

The majority of AFTNs are nontoxic as alluded to above. Most patients present due to cosmetic reasons, compressive symptoms or fear of cancer.

In the diagnosis and management of AFTN, there are two main issues. The first is that that the diagnosis is not always made as most patients with AFTNs are functionally 
euthyroid. The other is the paucity of data in the literature to offer evidence-based individualized management for patients.

\section{Aetiology}

The current theory on the development of hyperfunctioning nodules is the constitutive activation of Thyroid Stimulating Hormone Receptor (TSHR) due to a somatic point mutation and mutations of Gsa [5]. They are gain-of-function mutations. Pathophysiologically, the development of somatic mutations, progression in nodular growth and achieving autonomic activity has a strong connection to Iodine deficiency [6]. Small clones of cells with TSHR may grow to overcome inhibition by paracrine inputs; achieving an autonomic state [7]. The incidence of these mutations ranges from $8 \%-80 \%$ [5]. The absence of TSHR and Gsa mutations was noted in some AFTNs pointing out that further assessment of molecular pathways in AFTN is required [5].

On perusal of the literature, AFTNs have been described in some patients with Acromegaly. Exposure to long-term surges of growth hormone has been postulated as the possible reason for this.

\section{Outcome of AFTNs}

The progression of AFTN is variable. Outcomes can be categorized on activity (function), morphology, local effects of the nodule and pathological (possibility of malignancy) nature of the AFTN.

As mentioned earlier, about 10\% - 30\% may become 'hot/ toxic' nodules, but the majority will stay as 'cold/ nontoxic' nodules [4]. A nontoxic nodule may become toxic between 1 -11.8 years.

This may be one of the reasons why more toxic AFTNs were detected in the elderly [3]. The conversion probability is higher in larger nodules. Nodules larger than $3 \mathrm{~cm}$ are said to be more likely to be toxic $[3,4]$. The annual conversion rate is around $4 \%$ [8].

These facts signify the importance of close follow up of a non-toxic AFTN, clinically and biochemically after initial diagnosis.

Hot nodules may have variable activity levels demonstrating subclinical hyperthyroidism, overt thyrotoxicosis and elevated T3. Subclinical hyperthyroidism is the common outcome of most hot nodules. Our patient had subclinical hyperthyroidism with a $5 \mathrm{~cm}$ AFTN. She underwent left hemithyroidectomy due to gross deviation of the trachea to the right (Figure 2) and compressive symptoms. A lesser percentage of these nodules become thyrotoxic (especially nodules $>3 \mathrm{~cm}$ ) and commonly suppress extranodular normal gland activity which may render them invisible with low uptake of tracer at scintigraphy as in this patient.

An interesting biochemical finding in some AFTN is the presence of high T3. The likely explanation of the high T3 is an increase in primary production from the gland or peripheral conversion. High T3 has been demonstrated in both cold and hot nodules. Our patient initially presented with high T3 and normal T4 levels.

The pathological basis behind an AFTN being cold is the poor iodination of thyroglobulin due to decreased capacity of iodide transport. They retain the ability to organify iodide and may become hyperfunctioning/hot with time. This transition is hypothesized to happen in periods of excess iodine intake [9].

Morphologically an AFTN may remain the same, increasing or decreasing in size [10]. Some studies have noted the disappearance of the nodule as well [11]. Growing nodule compressing on the blood supply of its own has been postulated as the possible reason. Transient thyrotoxic events may occur following acute haemorrhagic infarction of an AFTN [3].

AFTNs are essentially benign conditions. Primary malignancy in the AFTN or the gland with toxic AFTN [12] is a concern but it is rare [13]. Studies have shown the incidence of malignancy in AFTN to be around 3\% [13]. Most malignancies are papillary or follicular in origin. $\mathrm{H}$ ürthle cell carcinoma has also been reported in some patients [14].

AFTN is very rare in children with incomplete data on malignancy risk [15]. In a child with a solitary thyroid nodule, a primary malignancy should always be excluded [16].

There is a consensus that hyperfunctioning thyroid nodules are rarely malignant [13]. Biopsy of hyperfunctioning nodules is not done routinely. Hence a hyperfunctioning thyroid carcinoma may masquerade as an AFTN. Studies have shown that around $50 \%$ of malignancies in toxic nodules are follicular carcinomas [17]. The size of the lesion, rapidity of growth, ultrasonic features and response to conventional treatment help arrive at a definitive diagnosis. As the majority are follicular lesions FNAC would not provide histological confirmation of malignancy and a hemithyroidectomy is required to confirm the diagnosis. If RAI was the primary treatment a non-responsive nodule (concerning thyrotoxicosis) would indicate the possibility of malignancy [17]. 


\section{Diagnosis}

As there are no tissue diagnostic criteria for AFTN; clinical, biochemical and radiological assessments are done to arrive at a diagnosis. The main goal is to establish TSH independent nodular hyperfunction.

Complete thyroid profile of TSH, fT4 and fT3 should be performed. TSH per se as a screening tool to diagnose AFTN is less sensitive. A recent meta-analysis has demonstrated $50 \%$ of scintigraphy proven AFTNs have a normal TSH [18]. Free T3 is paramount as low TSH and normal free T4 (fT4) is a frequent finding as in this patient.

An ultrasound scan (USS) of the thyroid will commonly reveal a single thyroid nodule with benign characteristics. Ultrasonically, suspicious features like microcalcifications are present in $50 \%$ AFTNs, invariably leading to FNAC to exclude a malignancy [19]. USS would also help morphological differentiation of an AFTN from a diffuse or a multinodular goitre and delineate cervical lymphadenopathy suggestive of a different diagnosis.

Subclinical or overt hyperthyroidism should indicate the need for scintigraphy. Even with normal TSH, scintigraphy is recommended in the investigation of a solitary nodule of thyroid exceeding $1-1.5 \mathrm{~cm}$ [20]. It will confirm the hyperfunctioning nodule and suppression of extranodular gland activity. This latter point is essential in differentiating an AFTN from Graves [15]. In Graves' background activity is present on scintigraphy. Both these entities can coexist and are known as Marine - Lenhart syndrome [21]. Such patients may need scintigraphy following suppressive thyroxine therapy to demonstrate the AFTN. The use of SPECT/CT fusion imaging is an upcoming imaging modality with higher sensitivity and specificity on diagnosing AFTN [22]. Apart from the radiation-induced complications in a nuclear medicine intervention, there are several pitfalls of scintigraphy concerning the assessment of AFTNs. Less than $1 \mathrm{~cm}$ cold nodules may be missed and posteriorly placed nodules may be displayed 'warm' (falsely) due to superimposed thyroid tissue. Some nodules are discordant and may fail to organify 123 I (preferred tracer) and fail to show the activity on scintigraphy. As mentioned earlier, cytological diagnosis is inconclusive. It may help exclude a malignancy, which is rare on an AFTN.

\section{Treatment}

A cold nodule may be left alone with periodic examination for an increase in size (ultrasonography +/- FNAC) and conversion to a toxic nodule (biochemical thyroid profile). They may undergo surgery due to other reasons such as poor cosmesis, compressive symptoms or suspicion of malignant conversion. Prophylactic treatment for cold nodules is not advocated [23]. However, levothyroxine therapy for cold nodules had been studied with debatable results. Exogenous T4 will suppress TSH, resulting in a lack of nodular growth and subsequent shrinkage [24].

Hypersecretory nodules need treatment to relieve the patient of symptoms as well as avoid cardiovascular and bony complications of untreated hyperthyroidism. Hot nodules will respond to pharmacological measures like anti-thyroid drugs (ATD), but toxicity will invariably recur once the treatment is stopped [25]. The only place for ATD therapy in the management of AFTN is to make a patient euthyroid in preparation for surgery or radioiodine 131I ablation (RIA).

The definitive treatment for a hot nodule would be surgery, RIA and minimally invasive therapies (MIT) like percutaneous ethanol injection therapy (PEIT), laser ablation (LA) or radiofrequency ablation (RFA). MITs are mainly useful in dealing with smaller AFTNs [26]. In general, multiple sessions are required to achieve acceptable results. The way forward would be to make a patient-tailored approach assessing pros and cons along with the availability of resources.

Surgical management minimally requires a hemithyroidectomy (lobectomy + isthmusectomy). The enucleation of AFTN is inadequate [23]. Swift resolution of hyperthyroidism is achieved yet should be balanced against the welldocumented complications of thyroidectomy. Post-treatment hypothyroidism has not been described of and recurrence is unlikely. Total thyroidectomy (TT) is not indicated in an AFTN as it is a solitary benign lesion. TT carries the risk of complications of total thyroidectomy in addition to lifelong thyroxine therapy.

Radioiodine ablation is suitable in all nodules, preferably $<5 \mathrm{~cm}$. The relative ease of therapy should be weighed against radiation-induced side effects and treatment failures. Following RIA treatment, hyperthyroidism will take months to settle. Studies have shown that at 1 year following RIA, development of hypothyroidism is less than $20 \%$ and recurrence of hyperthyroidism at 3\% [27]. Patient-based careful dose calculation may produce a more favourable outcome [28].

PEIT is a safe method when guided with USS colour Doppler. Exacerbation of symptoms, a significant number of recurrences $(35 \%)$ and temporary vocal cord paralysis have been reported [27]. Furthermore, resistance to diffusion of ethanol in solid AFTNs and ethanol leakage-induced pain renders its use limited. It also has been shown to cause periglandular fibrosis making subsequent surgery difficult [29]. 
USS guided thermal ablation techniques like LA and RFA have shown to be effective as solo therapies in small AFTNs. In larger AFTNs, thermal ablation techniques in tandem with RIA produce a quicker resolution of hyperthyroidism than RIA alone [30]. Post-procedural pain, haematoma formation and transient vocal cord paralysis have been reported [29].

\section{Salient points}

- AFTNs are rare but identification and treatment especially in subclinical hyperthyroidism are essential in avoiding toxicity-related complications.

- Thyroid scintigraphy is the standard diagnostic technique.

- Surgical and non-surgical therapies should be applied in a patient-tailored manner.

- Newer techniques and further study will enable clinicians of the future to offer evidence-based individualized treatment for most patients

- The treatment will be curative.

All authors disclose no conflict of interest. The study was conducted in accordance with the ethical standards of the relevant institutional or national ethics committee and the Helsinki Declaration of 1975, as revised in 2000 .

\section{References}

1. Taylor PN, Albrecht D, Scholz A, Gutierrez-Buey G, Lazarus JH, Dayan CM, et al. Global epidemiology of hyperthyroidism and hypothyroidism. Nat Rev Endocrinol. 2018;14(5):301-16. https://doi.org/10.1038/nrendo.2018.18

2. Goetsch E. Newer methods in the diagnosis of thyroid disorders: pathological and clinical. NY State J Med. 1918;18:259.

3. Hamburger JI. Evolution of toxicity in solitary nontoxic autonomously functioning thyroid nodules. J Clin Endocrinol Metab. 1980 Jun 1;50(6):1089-93.

https://doi.org/10.1210/jcem-50-6-1089

4. Pacini F, Chiofalo F, De Groot LJ. Thyroid Neoplasia. Vols 2-2, Endocrinology: Adult and Pediatric. 2015.

5. Krohn K, Führer D, Bayer Y, Eszlinger M, Brauer V, Neumann S, et al. Molecular pathogenesis of euthyroid and toxic multinodular goiter. Vol. 26, Endocrine Reviews. 2005. p. 504-24. https://doi.org/10.1210/er.2004-0005

6. Delange F. The disorders induced by iodine deficiency. Vol. 4, Thyroid. Mary Ann Liebert Inc.; 1994. p. 107-28.

https://doi.org/10.1089/thy.1994.4.107

7. Dawson T, Wynford-Thomas D. Does autocrine growth factor secretion form part of a mechanism which paradoxically protects against tumour development? Br J Cancer. 1995;71(6):1136-41. https://doi.org/10.1038/bjc.1995.222

8. Corvilain B. The natural history of thyroid autonomy and hot nodules. In: Annales d'Endocrinologie. 2003. p. 17-22.

9. Masini-Repiso AM, Cabanillas AM, Bonaterra M, Coleoni AH. Dissociation of thyrotropin-dependent enzyme activities, reduced iodide transport, and preserved iodide organification in nonfunctioning thyroid adenoma and multinodular goiter. J Clin Endocrinol Metab. 1994 Jul 1;79(1):39-44. https://doi.org/10.1210/jcem.79.1.8027249
10. Desforges JF, Mazzaferri EL. Management of a Solitary Thyroid Nodule. Vol. 328, New England Journal of Medicine. 1993. p. 553-9. https://doi.org/10.1056/NEJM199302253280807

11. Cheung PSY, Lee JMH, Boey JH. Thyroxine suppressive therapy of benign solitary thyroid nodules: A prospective randomized study. World J Surg. 1989 Nov;13(6):818-21.

https://doi.org/10.1007/BF01658447

12.Polyzos SA, Goulis DG. Coincidental thyroid papillary microcarcinoma in a patient treated for a toxic adenoma of the thyroid. Vol. 14, Archives of Iranian Medicine. 2011 p. 149-51.

13. Mirfakhraee S, Mathews D, Peng L, Woodruff SL, Zigman JM. A solitary hyperfunctioning thyroid nodule harboring follicular thyroid carcinoma: Case report and review of the literature. Endocr Rev. 2012;33(3). https://doi.org/10.1186/1756-6614-6-7

14. Yalla NM, Reynolds LR. Case Report. 2011;68-72. https://doi.org/10.4158/EP10334.CR

15. Ly S, Frates MC, Benson CB, Peters HE, Grant FD, Drubach LA, et al. Features and outcome of autonomous thyroid nodules in children: 31 consecutive patients seen at a single center. J Clin Endocrinol Metab. 2016 Oct 1;101(10):3856-62.

https://doi.org/10.1210/jc.2016-1779

16. Damle N, Gupta S, Kumar P, Mathur S, Bal C. Papillary carcinoma masquerading as clinically toxic adenoma in very young children. J Pediatr Endocrinol Metab. 2011 Dec 1;24(1112):1051-4. https://doi.org/10.1515/JPEM.2011.206

17. Liu J, Wang Y, Da D, Zheng M. Hyperfunctioning thyroid carcinoma: A systematic review. Mol Clin Oncol. 2019;11(6):535-50. https://doi.org/10.3892/mco.2019.1927

18. Treglia G, Trimboli P, Verburg FA, Luster M, Giovanella L. Prevalence of normal TSH value among patients with autonomously functioning thyroid nodule. Eur J Clin Invest. 2015;45(7):739-44. https://doi.org/10.1111/eci.12456

19. Dirikoc A, Polat SB, Kandemir Z, Aydin C, Ozdemir D, Dellal $\mathrm{FD}$, et al. Comparison of ultrasonography features and malignancy rate of toxic and nontoxic autonomous nodules: a preliminary study. Ann Nucl Med. 2015 Dec 1;29(10):883-9.

https://doi.org/10.1007/s12149-015-1018-y

20. Corvilain B, Moreno-Reyes R, Kyrilli A, Lytrivi M, Bourmorck C, Chami R. Is there still a role for thyroid scintigraphy in the workup of a thyroid nodule in the era of fine needle aspiration cytology and molecular testing? Vol. 5, F1000Research. Faculty of $1000 \mathrm{Ltd} ; 2016$. https://doi.org/10.12688/f1000research.7880.1

21. Neuman D, Kuker R, Vendrame F. Case Report Marine-Lenhart Syndrome : Case Report, Diagnosis , and Management. 2018;2018. https://doi.org/10.1155/2018/3268010

22. The role of Tc-99m SPECT/CT fusion images in diagnosis of autonomously functioning thyroid nodules [Internet]. [cited 2020 May 1]. Available from:

http://jnm.snmjournals.org/content/54/supplement_2/1893.short

23.Meier DA, Hamburger JI. An Autonomously Functioning Thyroid Nodule, Cancer, and Prior Radiation: Case Report and Hypothesis. Arch Surg. 1971;103(6):759-61.

https://doi.org/10.1001/archsurg.1971.01350120123024

24. Yousef A, Clark J, Suhail AR. Thyroxine suppression therapy for benign, non-functioning solitary thyroid nodules: A qualityeffects meta-analysis. Vol. 8, Clinical Medicine and Research. 
Marshfield Clinic; 2010. p. 150-8.

https://doi.org/10.3121/cmr.2010.881

25.Huysmans DA, Corstens FH, Kloppenborg PW. Long-term follow-up in toxic solitary autonomous thyroid nodules treated with radioactive iodine. J Nucl Med. 1991;32(1):27-30.

26.Cesareo R, Naciu AM, Iozzino M, Pasqualini V, Simeoni C, Casini A, et al. Nodule size as predictive factor of efficacy of radiofrequency ablation in treating autonomously functioning thyroid nodules. Int J Hyperth. 2018 Jul 4;34(5):617-23. https://doi.org/10.1080/02656736.2018.1430868

27. Yano Y, Sugino K, Akaishi J, Uruno T, Okuwa K, Shibuya H, et al. Treatment of autonomously functioning thyroid nodules at a single institution: Radioiodine therapy, surgery, and ethanol injection therapy. Ann Nucl Med. 2011 Dec 5;25(10):749-54. https://doi.org/10.1007/s12149-011-0526-7
28.Ronga G, Filesi M, D'Apollo R, Toteda M, Di Nicola AD, Colandrea $\mathrm{M}$, et al. Autonomous functioning thyroid nodules and $131 \mathrm{I}$ in diagnosis and therapy after 50 years of experience: What is still open to debate? Clin Nucl Med. 2013;38(5):349-53. https://doi.org/10.1097/RLU.0b013e318286bbda

29.Sung JY, Baek JH, Jung SL, Kim JH, Kim KS, Lee D, et al. Radiofrequency ablation for autonomously functioning thyroid nodules: A multicenter study. Thyroid. 2015 Jan 1;25(1):112-7. https://doi.org/10.1089/thy.2014.0100

30.Pacella CM, Mauri G. Is there a role for minimally invasive thermal ablations in the treatment of autonomously functioning thyroid nodules? Vol. 34, International Journal of Hyperthermia. Taylor and Francis Ltd; 2018. p. 636-8.

https://doi.org/10.1080/02656736.2018.1462537 